\title{
Méthodologie De Production Des Données Cartographiques Sur Le Couvert Forestier En République Du Congo, De 2000 À 2012
}

\section{Saturnine Carine Milandou, PhD student}

Faculté des Sciences et Techniques, Département de Géosciences et Environnement, Université Marien N’GOUABI. BP 69, Brazzaville,

République du Congo

Laboratoire de Géomatique, Centre National d'Inventaire et d'Aménagement des Ressources Forestières et Fauniques (CNIAF), BP : 1375. Brazzaville,

République du Congo.

Chérubins Brice Ouissika, Ingénieur des eaux et forêts

Laboratoire de Géomatique, Centre National d'Inventaire et d'Aménagement des Ressources Forestières et Fauniques (CNIAF), BP : 1375. Brazzaville,

République du Congo.

\section{Hugues Goma Boumba' PhD student}

Centre de Recherche sur les Tropiques Humides (CRTH), Université Marien

N'GOUABI. Brazzaville, République du Congo

Rémi d'Annunzio, PhD

Remote sensing and GIS, UNREDD team- Forestry department, FAO, Rome, Italie.

Georges Claver Boundzanga, Coordinateur National REDD

Ministère de l'Economie Forestière (MEF) Brazzaville, République du Congo

\section{Dominique Nganga, Professeur titulaire}

Faculté des Sciences et Techniques, Département de Géosciences et Environnement Université Marien N'GOUABI. BP 69, Brazzaville, République du Congo.

Doi:10.19044/esj.2018.v14n32p154 URL:http://dx.doi.org/10.19044/esj.2018.v14n32p154

\begin{abstract}
A forest-based mapping data production study was conducted in the Republic of Congo as part of the Reducing Emissions from Deforestation and Forest Degradation (REDD) process including sustainable forest management, forest conservation, and forest degradation. biodiversity and increasing carbon stocks (REDD +). The purpose of this study was to produce a forest cover map and cover changes for the period 2000 to 2012, which meets the criteria of the
\end{abstract}


national definition of forest adopted by the Republic of Congo and the evaluation of forest areas. and losses. The map produced was based on the combination of forest map parameters developed prior to the adoption of the forest definition criteria. The 2000 and 2010 Landsat image segmentation was used to create polygons that respect the definition and in which the values of the underlying maps were injected, through a decision tree transcribed in the $\mathrm{R}$ language and implemented in conjunction with the tools Open Foris Tools Kit. The results indicate an overall accuracy of $90 \%$ of the map without stratification, with greater accuracy for the forest (86\%) than for the losses (73\%). The forest occupies 23,517,000 hectares, representing $69 \%$ of the national territory. The forest area lost during this period was estimated at about 145,356 hectares, which represents an annual average of 12,113 hectares $(0.052 \%)$. These results lead to the conclusion that the Republic of Congo is one of the countries with high forest cover and low deforestation.

Keywords: Deforestation, Forest, REDD +, Republic of Congo

\section{Résumé}

Une étude de production de données cartographiques liées à la forêt a été réalisée en République du Congo dans le cadre du processus de réduction des émissions liées à la déforestation et la dégradation des forêts incluant la gestion durable des forêts, la conservation de la biodiversité et l'accroissement des stocks de carbone (REDD+). Cette étude visait la production d'une carte de couverture forestière et des changements de couverture pour la période de 2000 à 2012, qui réponde aux critères de la définition nationale de la forêt adoptés par la République du Congo et l'évaluation des superficies forestières et des pertes. La carte produite a été basée sur la combinaison des paramètres des cartes forestières élaborées antérieurement à l'adoption des critères de définition de la forêt. La segmentation d'images Landsat de 2000 et 2010 a été utilisée pour créer des polygones qui respectent la définition et dans lesquelles ont été injectées les valeurs des cartes sous-jacentes, à travers un arbre de décision transcrit dans le langage $\mathrm{R}$ et implémenté en conjonction avec les outils Open Foris Tools Kit. Les résultats indiquent une précision globale de $90 \%$ de la carte hors stratification, avec une précision plus forte pour la forêt $(86 \%)$ que pour les pertes (73\%). La forêt occupe 23517000 hectares représentant $69 \%$ du territoire national. Les surfaces forestières perdues au cours de cette période ont été estimées à près de 145356 hectares, ce qui représente une moyenne annuelle de 12113 hectares (soit 0, 052\%). Ces résultats permettent de conclure que la République du Congo fait partie des pays à haute couverture forestière et faible déforestation.

Mots clés : Déforestation, Forêt, REDD+, République du Congo, 


\section{Introduction}

La République du Congo, située au cœur du bassin du Congo, deuxième massif forestier tropical mondial, présente un couvert forestier non uniforme qui varie en fonction de la densité de la population, de la qualité des infrastructures de transport, de la richesse des forêts, de l'historique de leur exploitation et de l'existence de zones urbaines (NERF, 2017). Comme partout dans la zone tropicale, la forêt du Congo n'échappe pas au processus de réduction de sa superficie à travers la déforestation et de dégradation des forêts. Ces deux facteurs d'origine anthropique par le changement d'usage des sols forestiers, sont identifiés comme contributeur aux émissions de gaz à effet de serre dont les taux annuels se situent entre 12 et $25 \%$, comme souligné dans plusieurs travaux (Tsayem et Forting 2004 ; Pearson et al., 2005 ; Stern, 2006 ; Denman, et al., 2007 ; GIEC, 2007 ; van der Warf et al., 2009 ; Nasi et al., 2008; Chenost et al., 2010).

La déforestation et la dégradation des forêts ont conduit à la mise en place d'une politique prônant la Réduction des Emissions de gaz à effet de serre (GES) dues à la Déforestation et à la Dégradation forestière intégrant la gestion durable des forêts, la conservation et le renforcement des stocks de carbone forestière (REDD+) (Zang et al., 2002; Karsenty et Pirard, 2007b ; CCNUCC, 2007b ; CCNUCC, 2010 ; Tsayem, 2011 ; Hewson et al., 2014 ; Tchatchou et al., 2015).

La REDD+, est l'une des solutions proposées pour amoindrir la crise globale de l'environnement, en sus de nombreuses conventions sur le climat et la biodiversité, dont la plus récente est l'accord de Paris (CCNUCC, 2015), avec un accent particulier sur le carbone, élément essentiel dans la compréhension des changements climatiques, (Ramade, 2005 ; Brown, 1997; Tsayem, 2009a et b).

Considérant l'étendue de ses surfaces forestières et consciente du rôle essentiel que jouent les forêts tropicales dans la régulation du climat et la conservation de la biodiversité (Pascal, 2003 ; Ramade, 2005 ; Karsenty et Pirard, 2007b), la République du Congo s'est engagée depuis l'année 2008 dans le processus REDD+ afin de contribuer à l'atténuation des changements climatiques (RPP, 2011).

Plusieurs études de suivi du couvert forestier par télédétection à l'échelle nationale ont été menées en République du Congo (FACET, 2012 ; GAF, 2013 ; GFC, 2013 ; BRL, 2014) qui ont montré une forte couverture forestière et un taux faible de déforestation. Cependant, ces études ont été réalisées antérieurement à l'adoption par le Congo, des critères définissant la forêt dans le cadre de REDD+ : superficie minimale de la forêt : 0.5 ha, hauteur minimale des arbres : 3 mètres ; couverture de la canopée : 30\% (CNREDD, 2014), tel qu'exigé par la Conférence des Parties (CCNUCC 7) pour l'établissement du niveau des émissions de référence pour les forêts comme le 
point de repère pour évaluer les résultats obtenus par le pays dans la mise en œuvre des activités REDD+ (CCNUCC, 2012 et 2013).

$\mathrm{Au}$ regard de ce qui précède, il est apparu nécessaire de réaliser une étude de suivi du couvert forestier en République du Congo pour la période allant de 2000 à 2012 avec des critères qui respectent la définition nationale. De manière spécifique, il s'est agi de produire une carte de base au processus REDD+, combinant les caractéristiques de trois produits cartographiques sur la forêt et les changements de forêt existants sur le pays, à travers un arbre de décision clair et compréhensible et la segmentation d'images satellites . En outre, une évaluation des superficies forestières et des pertes de forêt est proposée au cours de ce travail, afin de servir de base pour la mise en œuvre des activités REDD+.

\section{Matériel et méthodes}

\subsection{Zone d'étude}

La République du Congo située entre $4^{\circ} \mathrm{N}$ et $5^{\circ} \mathrm{S}$ de latitude et entre $11^{\circ} \mathrm{O}$ et $19^{\circ} \mathrm{E}$ de longitude fait partie intégrante du « bassin forestier du Congo ». Étendue sur $342000 \mathrm{~km} 2$, elle est limitée par le Cameroun et la République Centrafricaine au Nord, la République Démocratique du Congo (RDC) à l'Est, le Gabon à l'Ouest, l'enclave du Cabinda et s'ouvre sur l'océan Atlantique sur $170 \mathrm{~km}$ de côtes au sud (SCN, 2009). Ce positionnement naturel fait du Congo la principale porte d'entrée et de sortie de l'Afrique Centrale (Figure 1).

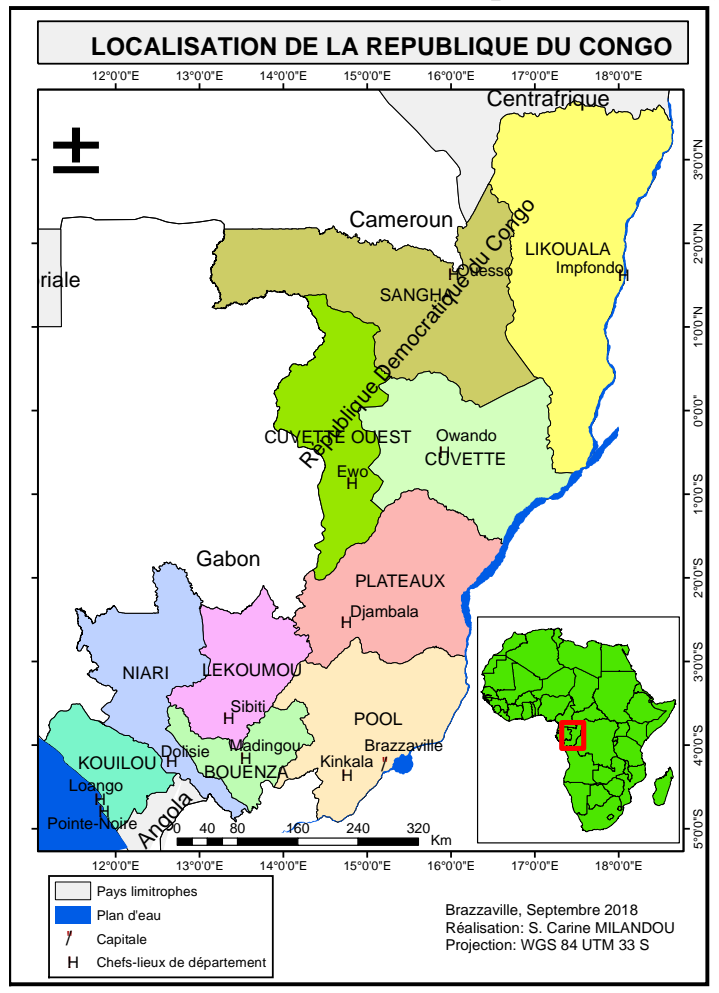

Figure 1 : Localisation du pays /Figure 1 : Location of the country 
La population en pleine croissance avoisine quatre millions d'habitants. La densité moyenne est de 10,5 habitants au $\mathrm{km}^{2}$ et le taux moyen annuel de croissance démographique est de 3,2\%.

\section{Climat}

Le climat est caractérisé par un régime pluviométrique variant selon que l'on se trouve au nord ou au sud du pays (Samba and al., 2007). Il est de type équatorial avec une structure bimodale dont la durée de la saison sèche diminue du Sud vers le Nord, 3 à 4 mois jusqu'à $2^{\circ} \mathrm{S}$ et 1 à 2 mois pour le reste du pays (SCN, 2009).

\section{Relief}

Les reliefs du Congo sont dans l'ensemble peu élevés, mais très variés. On peut reconnaître deux ensembles principaux de part et d'autre d'une ligne sudest - nord-ouest passant par Brazzaville et Zanaga : le Congo septentrional et le Congo sud-occidental, subdivisés eux-mêmes en unités plus petites (BRL, 2014).

\section{Pédologie}

Les sols du Congo sont en général acides, très sensibles à l'érosion hydrique et aux compactions mécaniques. Ils sont répartis en deux classes principales : les sols ferralitiques et les sols hydromorphes. Cependant, très localement, on observe aussi des sols d'érosion peu évolués sur des pentes très fortes, des podzols de nappe dans les vallées sableuses, des rendzines tropicales sur des collines de calcaires dolomitiques, et le long du littoral (Mapangui and Mpoundza., 2001 ; SCN, 2009).

\section{Végétation}

La végétation du Congo est caractérisée par des forêts et des savanes. La couverture forestière est très discontinue et hétérogène et comprend des forêts de terre ferme et des forêts inondées dans la cuvette congolaise. Elle se caractérise par une richesse biologique remarquable (BRL, 2014).

\subsection{Matériel et méthodes}

\subsubsection{Matériel de production cartographique}

Le traitement des données satellitaires et la production de la carte de changement d'occupation des sols ont été effectués avec les outils informatiques libres, gratuits et ouverts suivants OFGT 1.26 (2018), R 3.1.0 (R, 2018), QGIS 2.8 (QGis, 2018). L'ensemble de la chaîne de traitement a été implémenté dans un environnement de travail Linux (distribution Xubuntu 14.04) et est disponible en ligne (CNIAF, 2018). Par ailleurs, Collect Earth et Google Earth ont été utilisés pour l'analyse de la précision de la carte. 


\subsubsection{Données}

Les données utilisées dans ce travail sont composées des images satellitaires et des cartes forestières. Les images satellites sont de deux types : les images du capteur Landsat 7 ETM+ et les images SPOT. Les premières, avec une résolution spatiale de 30 mètres, sont des mosaïques téléchargées à partir du site du produit de Global Forest Change de l'Université du Maryland (Hansen et al. 2013) pour l'année 2000 et du site du projet WELD (World Enabled Landsat Dataset) pour l'année 2010 (WELD, 2018). De façon plus spécifique, les images de l'année 2000 sont sous forme de deux scènes dont le maillage est de 10 degrés, couvrant l'ensemble du Congo. Tandis que celles de 2010 sont composées de 28 scènes en format HDF en projection sinusoïdale couvrant tout le territoire du Congo. Toutes ces images (Landsat et Spot) ont été obtenues préalablement traité (géoréférencement, correction radiométrique, atmosphérique, etc...).

Les secondes issues du capteur SPOT, ont été acquises par l'intermédiaire du projet AFD-ASTRIUM Bassin du Congo et tirées de la base de données cartographiques du Centre National d'Inventaire et d'Aménagement des ressources Forestières et Fauniques (CNIAF). Au total 183 scènes d'une résolution spatiale de 10 mètres, constituant le pivot 2010 disponible pour le Congo, ont été exploitées pour l'analyse de la précision ou validation des pertes détectées.

Les cartes forestières quant à elles sont des données cartographiques sur la forêt et les changements de couverture forestière existant sur le pays. Ces données sont issues de la télédétection par analyse des données d'observation de la terre entre 2000 et 2012, dans le cadre de suivi du couvert forestier à l'échelle nationale. Ces données, élaborées antérieurement à l'adoption par la République du Congo des paramètres définissant la forêt, présentent chacune des caractéristiques qui leur sont spécifiques. Ces cartes sont :

La Carte forestière de GAF (2000 - 2010), élaborée à l'échelle nationale, dans le cadre de GSE Suivi forestier, Extension des Services REDD, projet MDDEF/REDD/GAF. La société GAF, connue sous le nom officiel de GAF AG, Munich, est une société Allemande qui a agi en tant que prestataire de services au Ministère du Développement Durable et de l'Economie Forestière (MDDEF) en République du Congo, dans le cadre de l'Extension GSE FM REDD (surveillance des forêts) pour la période 2000 à 2010.

La Carte forestière de Forêts d'Afrique Centrale évaluées par télédétection (FACET) - Atlas de la République du Congo- de 2000 à 2010, projet de l'Observatoire Satellital des forêts d'Afrique centrale (OSFAC) pour évaluer quantitativement, la dynamique spatio-temporelle des changements forestiers en Afrique Centrale à travers l'utilisation des données satellites multi-temporelles. Les résultats FACET sur le type, l'étendue et les pertes de couvert forestier sont une contribution utile pour plusieurs projets incluant le 
suivi de la biodiversité, la modélisation du climat, la conservation, la gestion des ressources naturelles, la planification de l'utilisation des terres, la Réduction des Émissions dues à la Déforestation et à la Dégradation des forêts (REDD). L'Atlas FACET est le résultat d'une étude sur l'étendue et les pertes brutes du couvert forestier en République du Congo de 2000 à 2010 (Atlas de la République du Congo, 2010).

Les deux cartes forestières (GAF et FACET) ont été obtenues à partir du Centre National d'Inventaire et d'Aménagement des Ressources forestières et fauniques (CNIAF).

La Carte forestière de Global Forest Change (GFC) de 2000 à 2012, est issue d'une étude globale par télédétection des changements de couvert arboré, menée par l'université du Maryland (Hansen et al., 2013). Des images Landsat de $30 \mathrm{~m}$ de résolution ont été analysées pour chaque année entre 2000 et 2012. Les arbres sont définis comme toute végétation ligneuse supérieure à $5 \mathrm{~m}$ de hauteur, et chaque pixel du produit contient une valeur de pourcentage de couverture et une indication sur le changement (perte, année de perte, gain). Les images de référence pour 2000 et 2012 sont les observations médianes en saison de végétation d'un jeu de données dont la qualité a été évaluée et validée. La couche disponible sur le site dédié (GFC, 2013) a été extraite et découpée aux frontières du Congo pour ce travail.

Les caractéristiques et les légendes de ces différentes cartes, potentiellement complémentaires et pertinentes pour le Congo, sont représentées respectivement par le tableau 1et les figure 2 et 3 ci-dessous :

Table 1 : Representation of the characteristics of the three cards/ Tableau 1 : Représentation des caractéristiques des trois cartes

\begin{tabular}{|c|c|c|c|c|c|c|c|}
\hline $\begin{array}{c}\text { Produit } \\
\text { cartographique }\end{array}$ & $\begin{array}{l}\text { Unité minimale de } \\
\text { cartographie (ha) }\end{array}$ & Dates & Classes & Précision & $\begin{array}{l}\text { Classe de } \\
\text { changement }\end{array}$ & $\begin{array}{l}\text { Taux annuel de } \\
\text { déforestation }\end{array}$ & Précision \\
\hline FACET & 0,36 & $\begin{array}{l}2000 \\
2005 \\
2010\end{array}$ & $\begin{array}{c}\text { Forêt primaire } \\
\text { Forêt secondaire } \\
\text { Forêt marécageuse } \\
\text { Non forêt }\end{array}$ & Non déterminée & Pertes & $0,07 \%$ & Non déternimée \\
\hline GFC & 0,09 & $\begin{array}{l}2000 \\
2012 \\
\end{array}$ & $\%$ couverture & $94 \%$ & \begin{tabular}{|c} 
Pertes par an \\
Gains
\end{tabular} & $0,08 \%$ & Non déterminée \\
\hline GAF & 1 & $\begin{array}{l}1990 \\
2000 \\
2010\end{array}$ & $\begin{array}{c}\text { Forêt } \\
\text { Non-Forêt }\end{array}$ & $97 \%$ & $\begin{array}{c}\text { Pertes + GIEC } \\
\text { Gains }\end{array}$ & $0,03 \%$ & $53 \%$ \\
\hline
\end{tabular}




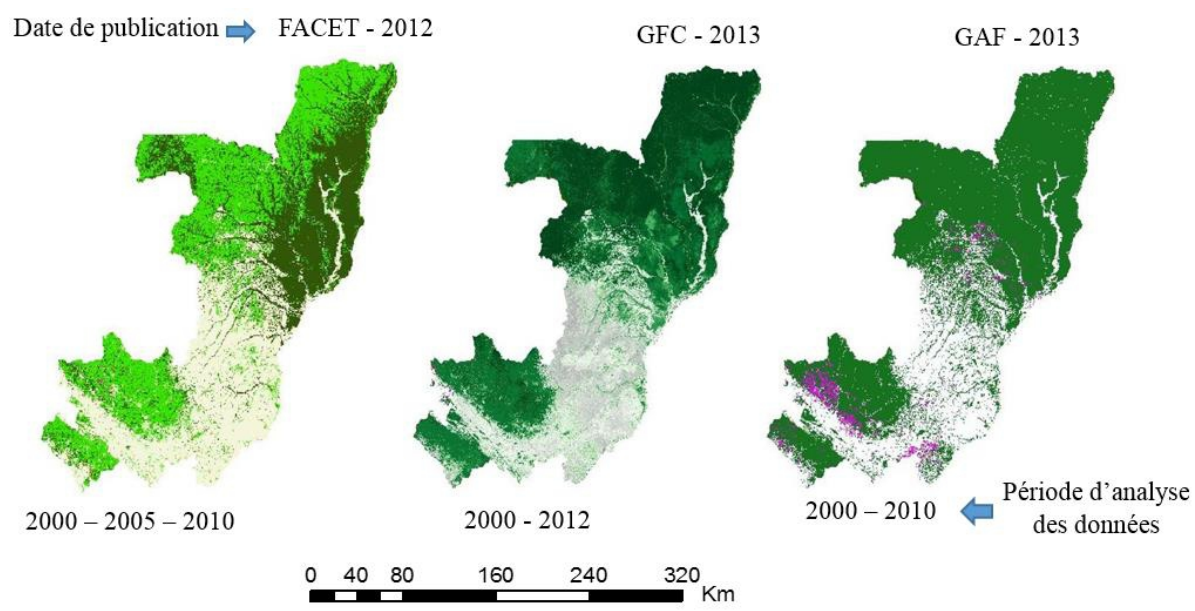

Figure 2. Representation of the three maps and their periods/ Figure 2. Représentation des trois cartes et leurs périodes
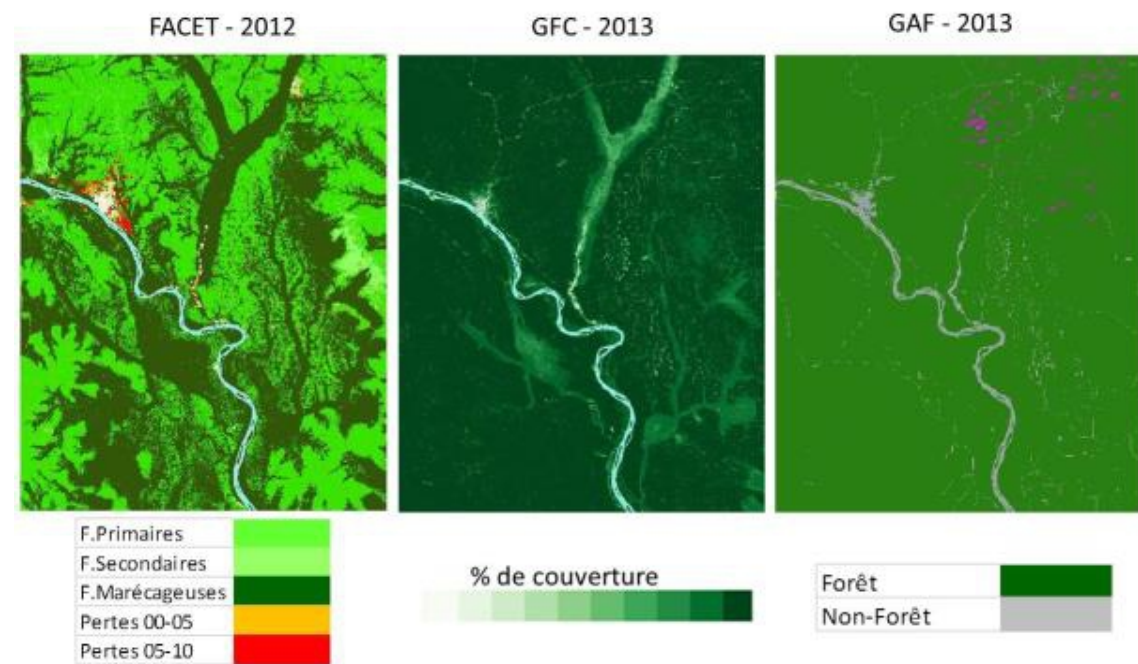

Figure 3. Illustration of forest maps and their legends in the Sangha area according to data from GAF, FACET and GFC/ Figure 3. Illustration des cartes forestières et leurs légendes dans la zone de la Sangha selon les données de GAF, FACET et GFC

\subsection{Méthode}

\subsubsection{Traitement des images et des cartes forestières}

Ce traitement a porté essentiellement sur la subdivision des deux scènes Landsat de 2000 téléchargées, pour correspondre à chacune des 28 scènes HDF de WELD. Ensuite, un composite à 6 bandes (bandes Rouge, Proche Infrarouge et Moyen Infrarouge pour 2000 et pour 2010) a été produit pour chaque scène et segmenté en polygones respectant une unité minimale de cartographie (MMU) de 0,5 hectare. La segmentation a été réalisée avec la fonction "oft-seg", en utilisant un algorithme d'inondation de bassins versants (watershed segmentation). 
Par ailleurs, pour chacune des cartes initiales, un code a été attribué à chaque classe (tableau 2) afin de faciliter l'intégration des informations dans les polygones de la segmentation en sortie, lors de la classification. Pour chaque polygone, les statistiques zonales des différents produits (GAF, FACET, GFC) ont été calculées en utilisant ces codes.

Table 2: Presentation of coded classes of cards /Tableau 2 : Présentation des classes codées des cartes

\begin{tabular}{|c|c|c|c|}
\hline $\begin{array}{c}\text { Produit } \\
\text { cartographique }\end{array}$ & \multicolumn{2}{|c|}{ Classes } & Codes \\
\hline \multirow{6}{*}{ FACET } & Forêt primaire & \multirow{3}{*}{ Forêt } & \multirow{3}{*}{1} \\
\hline & Forêt secondaire & & \\
\hline & Forêt marécageuse & & \\
\hline & \multicolumn{2}{|c|}{ Non forêt } & 2 \\
\hline & \multicolumn{2}{|c|}{ Pertes } & 3 \\
\hline & \multicolumn{2}{|c|}{$\mathrm{Eau}$} & 4 \\
\hline \multirow{6}{*}{ GFC } & $0-15 \%$ couverture & \multirow{2}{*}{ Non forêt } & \multirow{2}{*}{2} \\
\hline & $15-30 \%$ couverture & & \\
\hline & $30-60 \%$ couverture & \multirow{3}{*}{ Forêt } & \multirow{3}{*}{1} \\
\hline & $60-100 \%$ couverture & & \\
\hline & Gains & & \\
\hline & \multicolumn{2}{|c|}{ Pertes par an } & 3 \\
\hline \multirow{4}{*}{ GAF } & Forêt & \multirow{2}{*}{ Forêt } & 1 \\
\hline & Gains & & \\
\hline & \multicolumn{2}{|c|}{ Non-Forêt } & 2 \\
\hline & \multicolumn{2}{|c|}{ Pertes } & 3 \\
\hline
\end{tabular}

L'ensemble des données utilisées (les cartes forestières et des changements, images satellitaires) a été découpé pour les ajuster avec la limite du pays à partir des logiciels R et QGis.

\subsubsection{Extraction des couches cartographiques}

Dans la carte des changements de GAF, il a été constaté que la conversion (pertes) de la classe Forêt en Infrastructure entre 2000 et 2010, correspond en majorité à des routes qui sont des éléments linéaires, difficiles à détecter en segmentation automatique. Ces routes éditées manuellement avec une forte valeur ajoutée, ont été extraite afin de les intégrer dans la carte finale. De même, les pertes des années 2011 et 2012 du produit GFC ont aussi été extraites pour couvrir la période de l'étude (2000-2012). Cette extraction a été faite à partir d'un script écrit et exécuté dans le logiciel $\mathrm{R}$.

\subsubsection{Production cartographique}

\subsubsection{Arbre de décision et classification}

Un arbre de décision a été créé pour affecter les valeurs ou codes des cartes sous-jacentes dans les polygones issus de la segmentation du composite.

Sur la base des statistiques zonales des cartes pour chaque polygone, l'arbre de décision a été conçu en fixant un taux de représentation de $30 \%$ de 
chaque variable ou classe pour chaque carte, en considérant la concordance des classes dans au moins deux des trois cartes initiales. Pour une carte binaire donnée (forêt / non forêt, perte / non perte), le polygone est affecté à une classe si cette classe occupe plus de $30 \%$ du polygone. Par exemple dans le cas des pertes, pour un polygone donné, la valeur du polygone de sortie est affectée comme «Perte» si au moins deux produits sur trois (e.g. GFC et GAF) respectent les critères correspondant à des pertes. Cet arbre est complet et prend en compte toutes les combinaisons possibles des cartes d'entrée: GAF, FACET, GFC, mais aussi la stratification de FACET pour la forêt et les pertes, l'intégration des routes et des pertes extraites respectivement des produits GAF et GFC.

La classification a consisté à incorporer et à combiner les valeurs des cartes initiales dans la segmentation (polygones) à travers l'arbre de décision transparent et justifié transcrit dans le langage $\mathrm{R}$ et appliqué aux routines d'exécution développées dans l'environnement Open Foris Geospatial Toolkit (OFGT).

L'implémentation de l'arbre de décision a conduit à une couche cartographique préliminaire de 2000 à 2010 avec les classes de forêt, non forêt, pertes et eau. La classe de forêt a été discriminées sur la base de la stratification du produit FACET, suivant la règle de priorité ci-après : Secondaire $>$ Primaire $>$ Marécageuse. A la carte thématique générée ont été intégrées par-dessus l'arbre de décision, les routes extraites du produit de GAF avec écrasement au préalable des valeurs sous-jacentes. La classe des pertes a été combinée avec les pertes des années 2011 et 2012 extraites du produit GFC, après filtrage au préalable de tous les éléments de taille inférieure à 0,5 ha. L'ensemble de ces pertes a par la suite été stratifié en prenant pour chaque polygone la classe majoritaire de forêt sous-jacente. Ce processus a conduit à une carte thématique finale d'occupation du sol et de pertes de forêt de 2000 à 2012 dont les classes et codes sont présentés dans le tableau 3 ci-dessous.

Table 3. Different strata of the map and their codes/ Tableau 3. Différentes strates de la carte et leurs codes

\begin{tabular}{|l|l|}
\hline \multicolumn{2}{|c|}{ Carte stratifiée } \\
\hline Code & Classe \\
\hline 2 & Non-Forêt \\
\hline & \\
\hline 4 & Eau \\
\hline 11 & Forêt Primaire \\
\hline 12 & Forêt Secondaire \\
\hline 13 & Forêt Primaire / Marécageuse \\
\hline 31 & Pertes Forêt Primaires \\
\hline 32 & Pertes Forêt Secondaire \\
\hline 33 & Pertes Forêt Primaire / Marécageuse \\
\hline 34 & Pertes Forêt Primaire vers routes \\
\hline
\end{tabular}




\subsubsection{Analyse de la précision de la carte d'occupation du sol de 2000 - 2012}

Elle a consisté à comparer la classification obtenue avec une autre source de données considérée comme de plus haute qualité par rapport à celles utilisées dans la production de cette carte. La méthode mise au point par Olofsson et al., (2014) déclinée en trois étapes : la définition du système d'échantillonnage, l'adoption d'un système de réponse optimal et l'analyse, a été utilisée.

Un échantillonnage stratifié a été réalisé avec répartition des points aléatoires dans chaque strate. Au total 908 points distribués entre les classes ont été aléatoirement tirés, avec un minimum de 50 points par classe. L'échantillonnage des classes majoritaires a été fait en choisissant aléatoirement des points dans une grille (paquet raster et sample dans $\mathrm{R}$ ), tandis que l'échantillonnage des classes rares l'a été en utilisant la fonction raster To Points dans $\mathrm{R}$, puis la fonction sample.

Pour les classes majoritaires stables (Forêt et Non Forêt) le système de réponse choisi a été l'outil Collect Earth où les points sont visuellement interprétés en utilisant les images présentes dans Google Earth, Bing map et Heremap. Pour les classes de pertes, tous les points ont été évalués visuellement à partir de la comparaison d'images Landsat pour l'année 2000 et d'images SPOT pour l'année 2010. Les résultats sont présentés sous forme d'une matrice de confusion et une table des surfaces estimées corrigées pour les strates agrégées et la carte stratifiée. La précision globale de la classification et les superficies de chaque classe ont été estimées.

\section{Résultats}

\section{Segmentation d'images}

Plus de cinquante millions polygones respectant l'unité minimale de cartographie, sont issus de la segmentation d'images (composite). La production du composite a permis de mieux identifier les grands types de végétation. La figure 4 ci-dessous illustre la segmentation superposée avec le composite dans une zone spécifique du pays. 


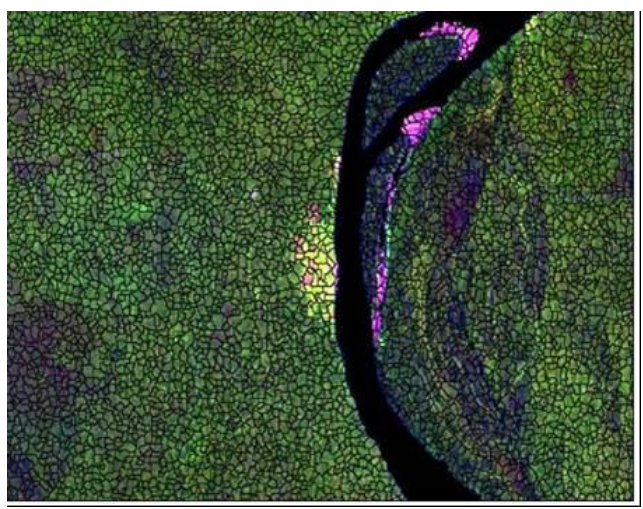

Figure 4: Illustration of a segmented image/ Figure 4: Illustration d'une image segmentée

\section{Arbre de décision}

L'arbre de décision a permis d'affecter aux polygones de sortie, les valeurs des rasters sous-jacentes. L'arbre, illustré par la figure (5), présente plusieurs branches $(\mathrm{Br})$ correspondant chacune à une classe en sortie. La première partie de l'arbre (B1 à B16) a conduit à une carte préliminaire avec quatre classes (forêt, non forêt, pertes et eau), laquelle a été stratifiée à partir de la deuxième partie de l'arbre. C'est-à-dire de la branche 16 à 18 (St 16-18) pour la forêt en forêt primaire, secondaire et marécageuse, et de la branche 19 à 21 pour les pertes. 


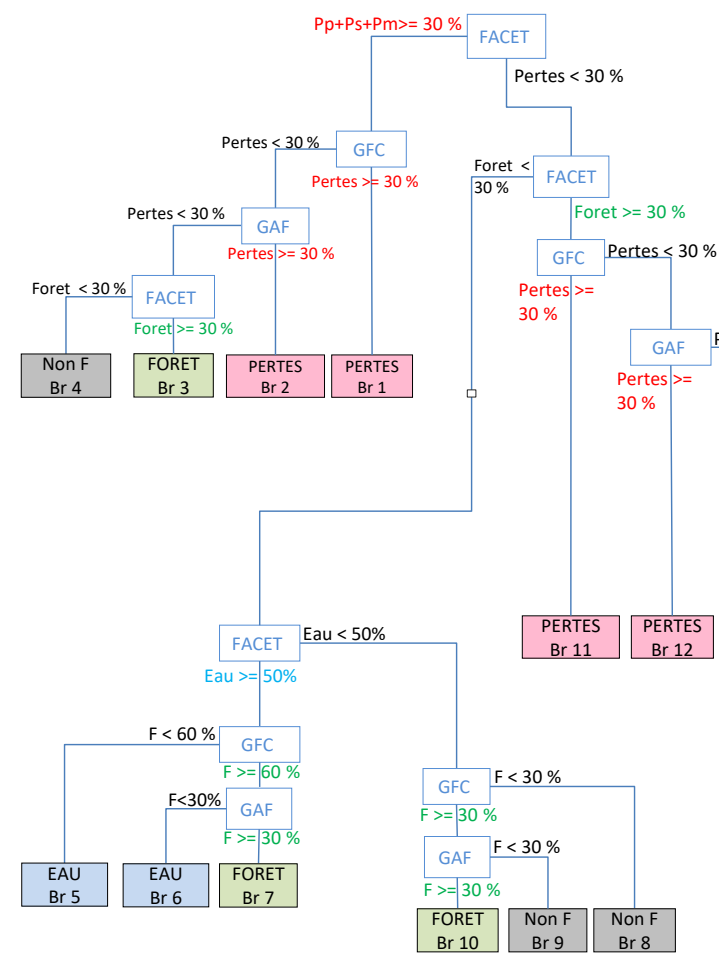

\section{Stratification}

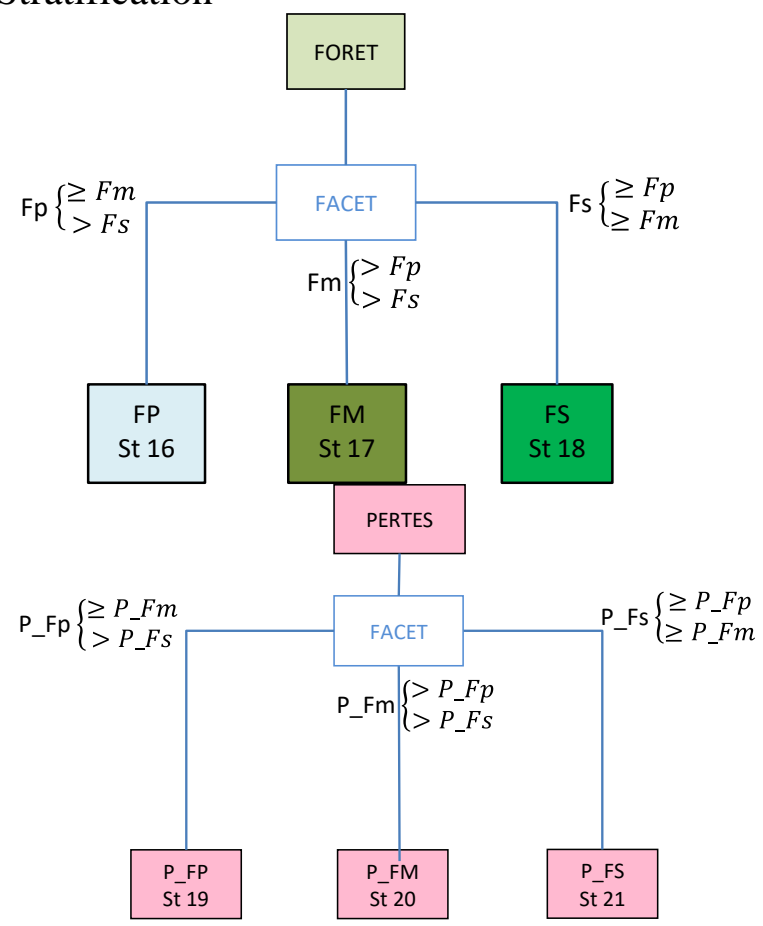

$\mathrm{Br}=$ Branche

P_Fp= Perte en forêt primaire

$P \_F s=$ perte en forêt secondaire $P$ Fm $=$ perte en forêt marécageuse $\mathrm{St}=$ stratification

Figure 5. Representation of the decision tree/ Figure 5. Représentation de l'arbre de décision 


\section{Fréquence d'apparition des branches}

L'analyse de la fréquence d'apparition des branches indique qu'elle diffère d'une branche à une autre selon l'information sous-jacente. Le tableau 4 ci-dessous donne un exemple d'analyse de la fréquence d'apparition de chaque branche pour une zone spécifique du Congo, (cas du département de la Likouala).

Table 4. Representation of the occurrence frequency of each branch in Likouala/ Tableau 4. Représentation de la fréquence d'apparition de chaque branche dans la Likouala

\begin{tabular}{cccc} 
branche & classe & polygone & prc \\
\hline 1 & 3 & 2696 & $0.17 \%$ \\
2 & 3 & 485 & $0.03 \%$ \\
3 & 1 & 1709 & $0.11 \%$ \\
4 & 2 & 445 & $0.03 \%$ \\
5 & 4 & 12302 & $0.76 \%$ \\
6 & 4 & 24 & $0.00 \%$ \\
7 & 1 & 11 & $0.00 \%$ \\
8 & 2 & 10029 & $0.62 \%$ \\
9 & 2 & 27718 & $1.72 \%$ \\
10 & 1 & 2575 & $0.16 \%$ \\
11 & 3 & 2764 & $0.17 \%$ \\
12 & 3 & 1993 & $0.12 \%$ \\
13 & 1 & 1547885 & $96.09 \%$ \\
14 & 1 & 100 & $0.01 \%$ \\
15 & 1 & 72 & $0.00 \%$ \\
16 & 4 & 109 & $0.01 \%$ \\
& & & \\
& TOTAL & 1610917 & $100.00 \%$
\end{tabular}

Avec classe 1 = Foret, classe $2=$ Non Foret, classe $3=$ Perte, classe $4=$ Eau

Le tableau 2 indique le nombre de polygones portant une variable donnée à la sortie, en fonction de la branche considérée. Ainsi sur 1610917 polygones, la configuration correspondant à la branche 3 de l'arbre apparaît 1709 fois, ou 72 fois pour de la branche 15 .

D'autre part, la très forte prédominance de la branche 13, correspond aux zones où FACET et GFC indiquent une couverture forestière supérieure à $30 \%$. Une illustration du résultat en sortie pour la branche 13 de l'arbre de décision et le script y afférent, est donnée ci-dessous par la figure 6 .

\section{\# Branche 13 -> forêt}

$(96.09 \%)$ 

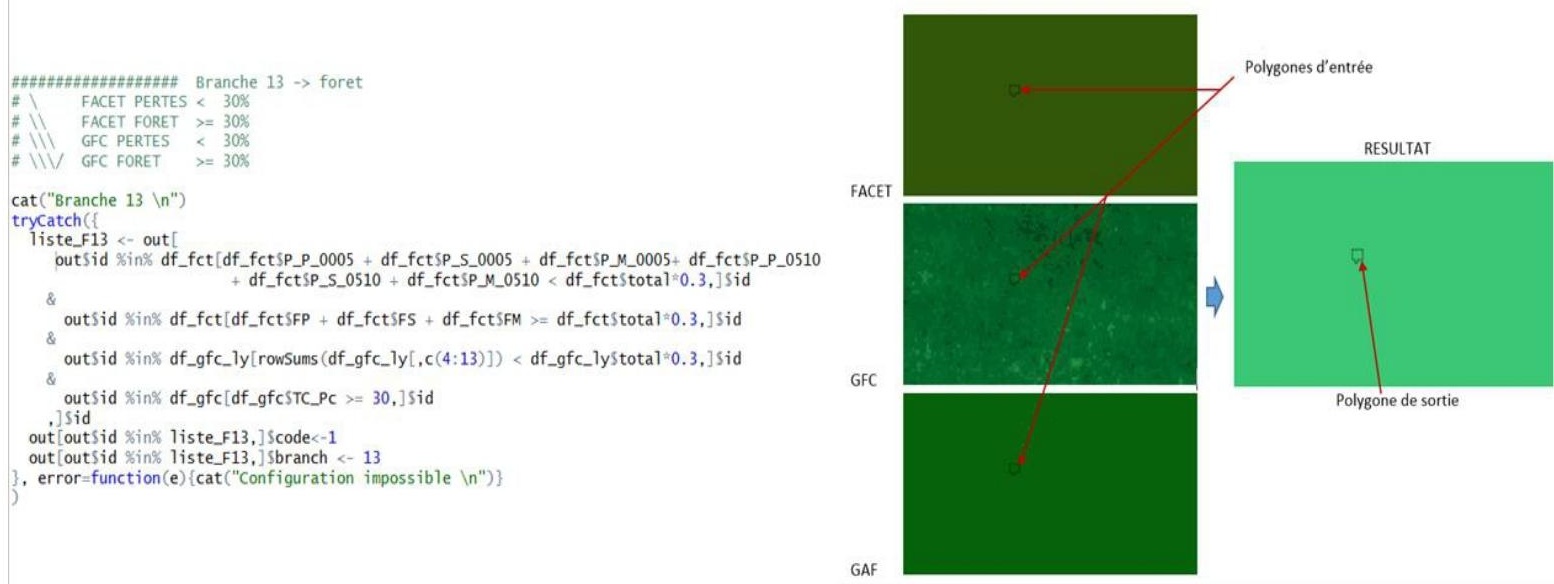

Figure 6: Illustration of branch 13 of the decision tree/ Figure 6: illustration de la branche 13 de l'arbre de décision.

Par contre, la stratification répartit les forêts et les pertes de la façon suivante dans cette zone (tableau 5):

Table 5. Distribution of forests and losses/ Tableau 5. Répartition des forêts et des pertes

\begin{tabular}{lcccc} 
& Forêt & Perte & \%Forêt & \%Perte \\
\cline { 2 - 5 } primaire & 413988 & 3204 & $26,67 \%$ & $40,36 \%$ \\
marécageuse & 1122117 & 762 & $72,28 \%$ & $9,60 \%$ \\
secondaire & 16247 & 3972 & $1,05 \%$ & $50,04 \%$ \\
total & 1552352 & 7938 & &
\end{tabular}

Ce tableau indique que les forêts marécageuses sont prédominantes mais qu'elles subissent peu de pertes, alors que les forêts secondaires concentrent sur moins de $1 \%$ de la superficie des forêts, la moitié des pertes enregistrées (les pertes ont lieu là où les forêts sont déjà dégradées).

\section{Produit cartographique : occupation du sol de 2000 à 2012}

La combinaison des caractéristiques des trois produits à travers l'arbre de décision, a abouti à une carte thématique d'occupation du sol et de pertes de forêt, en format Raster de $30 \mathrm{~m}$ de résolution spatiale. La figure 7 représente la répartition spatiale des différentes classes sur le territoire nationale, avec un agrandissement illustrant les classes dans des zones spécifiques. 

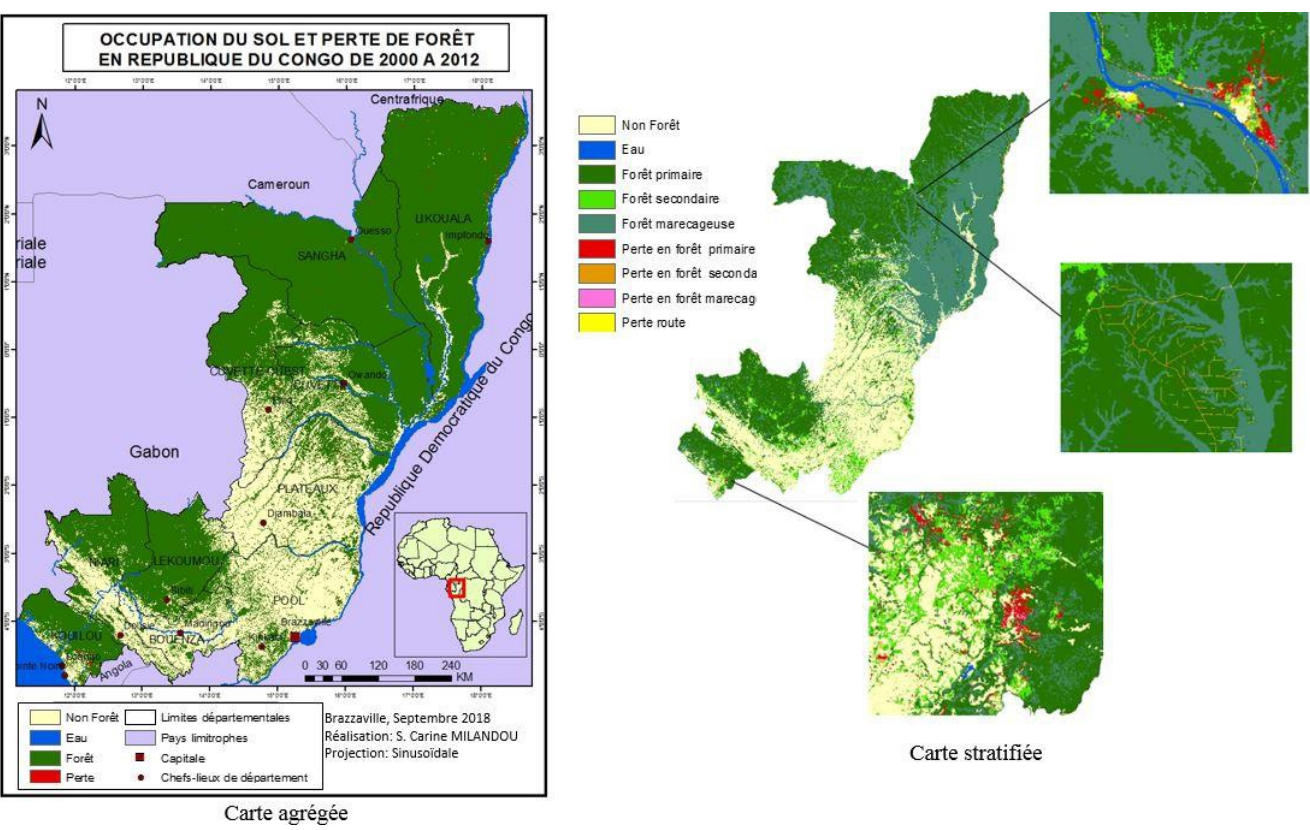

Figure 8. Map of Land Cover and Forest Loss 2000 to 2012/ Figure 8. Carte d'occupation du sol et de pertes de forêt de 2000 à 2012

L'analyse de cette carte montre que le territoire congolais est occupé en majorité par la forêt. Cette forêt, inégalement répartie, est plus importante dans la partie nord du pays.

\section{Analyse de la précision}

La classification a été évaluée par la matrice de confusion à travers le calcul de la précision globale de classification. Il ressort de cette analyse que la précision globale de classification est estimée à $90 \%$ pour les classes agrégées, avec une précision plus élevée pour les classes stables notamment $91 \%$ pour la forêt et $86 \%$ pour la non forêt, que pour les pertes $73 \%$ (tableau 6). Les précisions producteur et utilisateur élevées pour les classes majoritaires (forêt et non forêt), sont comprises entre 80 et $94 \%$, indique une bonne coïncidence des classes de la carte avec les données du terrain (de référence).

Table 6: Confusion Matrix for Aggregated Classes/ Tableau 6 : Matrice de confusion pour les classes agrégées

\begin{tabular}{|c|c|c|c|c|c|}
\hline & Forêt & $\begin{array}{c}\text { Non } \\
\text { Forêt }\end{array}$ & Pertes & Total & Précision Utilisateur \\
\hline Forêt & $\mathbf{4 1 9}$ & 40 & 0 & 459 & $91 \%$ \\
\hline Non Forêt & 29 & $\mathbf{1 8 2}$ & 0 & 211 & $86 \%$ \\
\hline Pertes & 47 & 7 & $\mathbf{1 4 5}$ & 199 & $73 \%$ \\
\hline Total & 495 & 229 & 145 & 869 & \\
\hline $\begin{array}{c}\text { Précision } \\
\text { Producteur }\end{array}$ & $94 \%$ & $80 \%$ & $100 \%$ & \\
\hline Précision globale & & & \multicolumn{7}{|c|}{$\mathbf{9 0 \%}$} \\
\hline
\end{tabular}


A propos de la matrice stratifiée, le tableau 7 montre que la précision globale baisse à $60 \%$ faisant suite à la confusion entre certaines strates. L'exemple est donné par la strate de forêt marécageuse qui a présenté des confusions essentiellement avec la strate forêt primaire. Néanmoins, les classes stables ont été mieux identifiées, avec respectivement $86 \%$ pour la non forêt et $73 \%$ pour la forêt primaire. Ces classes sont les plus représentées sur le territoire national.

Table 7. Class confusion matrix of the map/ Tableau 7. Matrice de confusion des classes de la carte.

\begin{tabular}{|c|c|c|c|c|c|c|c|c|c|c|c|}
\hline & nf & eau & fp & fs & fm & pp & ps & pm & pr & total & prec_uti \\
\hline Non forêt & 142 & 1 & 6 & 14 & 3 & 0 & 0 & 0 & 0 & 166 & $86 \%$ \\
\hline Eau & 9 & 30 & 1 & 0 & 5 & 0 & 0 & 0 & 0 & 45 & $67 \%$ \\
\hline Forêt primaire & 7 & 0 & 139 & 33 & 12 & 0 & 0 & 0 & 0 & 191 & $73 \%$ \\
\hline Forêt secondaire & 26 & 0 & 40 & 30 & 3 & 0 & 0 & 0 & 0 & 99 & $30 \%$ \\
\hline Forêt marécageuse & 7 & 0 & 100 & 19 & 43 & 0 & 0 & 1 & 0 & 170 & $25 \%$ \\
\hline Perte primaire & 0 & 0 & 7 & 4 & 0 & 22 & 12 & 2 & 3 & 50 & $44 \%$ \\
\hline Perte secondaire & 3 & 0 & 10 & 6 & 0 & 2 & 27 & 2 & 0 & 50 & $54 \%$ \\
\hline Perte marécageuse & 1 & 1 & 7 & 3 & 3 & 2 & 10 & 22 & 1 & 50 & $44 \%$ \\
\hline Perte route & 2 & 0 & 5 & 2 & 0 & 2 & 10 & 0 & 28 & 49 & $57 \%$ \\
\hline $\begin{array}{c}\text { précision } \\
\text { producteur }\end{array}$ & $86 \%$ & $49 \%$ & $56 \%$ & $11 \%$ & $72 \%$ & $88 \%$ & $49 \%$ & $7 \%$ & $82 \%$ & & \\
\hline Précision globale & & & & & & $60 \%$ & & & & & \\
\hline
\end{tabular}

\section{Estimation des superficies}

Les tableaux 8 et 9 représentent respectivement les superficies des classes agrégées et de la carte stratifiée.

Table 8. Accuracy Analysis Results for Aggregated Classes /Tableau 8 Résultats de

l'analyse de la précision pour les classes agrégées

\begin{tabular}{|c|c|c|c|c|c|c|}
\hline \multirow[b]{2}{*}{ Classe agrégée } & \multicolumn{3}{|c|}{ Superficie (x1000 ha) } & \multirow[b]{2}{*}{ Proportion } & \multirow[b]{2}{*}{$\begin{array}{l}\text { Précision } \\
\text { utilisateur }\end{array}$} & \multirow[b]{2}{*}{$\begin{array}{l}\text { Précision } \\
\text { Producteur }\end{array}$} \\
\hline & $\begin{array}{c}\text { Avant } \\
\text { correction }\end{array}$ & $\begin{array}{c}\text { Après } \\
\text { correction }\end{array}$ & IC à $95 \%$ & & & \\
\hline Forêt & 24326 & 23517 & 778 & $68,9 \%$ & $91 \%$ & $94 \%$ \\
\hline Non Forêt & 9673 & 10464 & 772 & $30,7 \%$ & $86 \%$ & $80 \%$ \\
\hline Pertes & 127 & 145 & 104 & $0,4 \%$ & $73 \%$ & $100 \%$ \\
\hline Total & 34127 & 34127 & & & $90 \%$ & \\
\hline
\end{tabular}

L'occupation du sol par la forêt représente 23517000 ha (+- 778,000 ha), soit $69 \%$ du territoire national. Elle représente la strate dominante à l'échelle nationale, suivie de la strate de non forêt, qui regroupe les agglomérations et l'ensemble des savanes, avec 10464000 ha (+- 772 000h). Cependant, la stratification montre une variation dans la répartition des différentes catégories de forêt sur l'ensemble du pays (tableau 9). 
Table 9: Results of precision analysis for stratified classes/ Tableau 9: Résultats de l'analyse de la précision pour les classes stratifiées

\begin{tabular}{|c|r|r|r|c|}
\hline Classe & \multicolumn{2}{|c|}{ Superficie (x1000 ha) } & \\
\hline Nont Foret & \multicolumn{1}{c|}{$\begin{array}{c}\text { Après } \\
\text { correction }\end{array}$} & IC à 95\% & Proportion \\
\hline Eau & 9590 & 10341 & 759 & $30 \%$ \\
\hline Foret $\mathbf{P}$ & 12364 & 123 & 123 & $0 \%$ \\
\hline Foret $\mathbf{S}$ & 1686 & 4459 & 1234 & $46 \%$ \\
\hline Foret $\mathbf{M}$ & 10277 & 3498 & 894 & $13 \%$ \\
\hline Perte $\mathbf{P}$ & 52 & 41 & 8 & $0 \%$ \\
\hline Perte $\mathbf{S}$ & 36 & 62 & 9 & $0 \%$ \\
\hline Perte $\mathbf{M}$ & 11 & 13 & 4 & $0 \%$ \\
\hline Perte $\mathbf{R}$ & 28 & 30 & 6 & $0 \%$ \\
\hline Total & 34127 & 34127 & & \\
\hline
\end{tabular}

Quant aux pertes, environ 145000 hectares de forêt ont été perdus entre 2000 et 2012. Ce qui représente une moyenne annuelle de 12113 hectares et un taux moyen de perte annuelle estimé à $0,052 \%$.

\section{Discussion}

\section{Approche méthodologique}

L'approche méthodologique pour la production de la carte d'occupation du sol et l'évaluation des pertes de forêt basée sur la combinaison des données existantes à travers un arbre de décision est une première en République du Congo. Les outils gratuits et libres utilisés offrent une indépendance de la méthodologie vis-à-vis d'une licence. Cette méthodologie, basée sur la télédétection est reproductible en exécutant des scripts (CNIAF, 2018).

L'utilisation de la télédétection est recommandée pour l'évaluation des données cartographiques historiques pour l'établissement des scénarios de référence et permet aux systèmes nationaux de monitorage d'évaluer et de suivre l'évolution des surfaces forestières et des surfaces déforestées (Tsayem, 2011 ; FAO, 2013 ; GFOI, 2016).

La taille minimale des polygones issus de la segmentation d'images ( 0,5 hectare) et les différentes branches de l'arbre établies sur la base d'un taux de représentation des classes des produits sous jacents, pour définir la variable de sortie, sont en accord avec les critères définissant la forêt dans le cadre de REDD+ en République du Congo. Ce qui signifie que ces résultats sont cohérents et conformes aux exigences de la CCNUCC, notamment la décision 16/CMP.1 qui stipule que les Parties visées à l'annexe I retiennent, aux fins de l'application de la définition des «forêts» donnée dans la décision 16/CMP.1, une seule et unique valeur minimale comprise entre 10 et $30 \%$ pour 
le couvert du houppier, entre 0,05 et 1 hectare pour la superficie et entre 2 et 5 mètres pour la hauteur des arbres.

De la même manière, la période de référence de 2000 à 2012 est couverte à travers l'intégration de la couche des pertes extraites du produit GFC (2013), en prenant pour chaque polygone la classe majoritaire de forêt sous-jacente, après filtrage de tous les éléments de taille inférieure à 0,5 hectare.

Les résultats de cette étude respectent les critères de crédibilité pour les données d'activités relatives à l'établissement des niveaux d'émissions de référence pour les forêts (GIEC, 2006 ; FAO, 2015 ; GFOI, 2016).

\section{Carte d'occupation du sol}

La carte thématique d'occupation du sol est un raster de 30 mètres de résolution spatiale. Elle est de projection sinusoïdale. Comme l'indique Snyder (1989), cette projection est conforme et respecte les superficies. En outre, elle subit le minimum de déformation en son centre, ce qui convient particulièrement pour le Congo (fig.4). C'est la projection native des images HDF utilisées pour la mosaïque des images Landsat 2010 et le découpage en boites.

La définition des classes thématiques dans ce produit est basée sur la stratification dans le produit FACET (2010), où la Forêt est définie comme un espace d'au moins 0,5 hectare de superficie, avec des arbres d'au moins trois (3) mètres de haut et d'une couverture de la canopée d'au moins $30 \%$ à une résolution spatiale de $30 \mathrm{~m}$.

Les différentes catégories de forêts dans ce produit se situent dans l'intervalle des classes forestières en Afrique centrale identifiées par télédétection, dans diverses cartes de végétation à l'échelle régionale et locale (Laporte et al., 1995; Achard et al., 2002 ; Mayaux et al., 1997 ; Mayaux et al., 2003; FRA, 2010; De Wasseige et al., 2012 et Verhegghen et Defourny, 2010; Verhegghen et al., 2012 ).

Les précisions globales de la classification estimées à $90 \%$ pour les classes agrégées et $60 \%$ pour la stratification sont acceptables, car selon Congalton (1991), une classification est jugée acceptable lorsque la précision globale avoisine $80 \%$. Ce qui sous - entend cependant, que la grande précision cartographique obtenue lorsque l'on considère la carte agrégée, est due au nombre réduit des classes, comme l'ont indiqué Caloz et al., (2001). Ces précisions se rapprochent des travaux réalisés par d'autres auteurs tels que Akadje et al., (2014) et Adjonou et al., (2010) et Bouetou-Kadilamio et al, (2017).

L'analyse de la matrice de confusion présente une bonne identification aussi bien des classes stables (forêt et non forêt) que des pertes. Cependant, pour la discrimination de la strate forêt, des grandes confusions d'interprétation 
ont été notées entre les classes de forêt primaire et marécageuse. Ce qui a entrainé une sous estimation de la forêt marécageuse.

La superficie totale forestière estimée à 23.517.000 ha dans cette étude avoisine celle indiquée dans de nombreux travaux de cartographie forestière (Mayaux et al., 2003 ; De Wasseige et al., 2009, 2012 et 2014, et Verhegghen et Defourny, 2010).

L'analyse des pertes de forêt montre un faible taux annuel de l'ordre de $0,052 \%$. Il est essentiel de remarquer qu'aucun des produits initiaux utilisés ne respecte la définition des forêts au Congo (MMU de 0,5ha), avec pour conséquence une potentielle surestimation (FACET / GFC avec une MMU inférieure) ou sous-estimation (GAF avec une UMM supérieure) des pertes réelles de forêt au Congo, évaluées dans cette étude, à 12113 hectares par an. Néanmoins, ce taux est inférieur à celui des travaux d'Ernst et al. (2010) et De Wasseige et al (2009), évalué respectivement à $0,16 \%$ entre 2005 et 2010 et 0,07\% en 2010, en République du Congo. Ces pertes sont enregistrées dans toutes les catégories de forêt avec une dominance dans la forêt secondaire (tableau 8). Toutefois, il sied de relever que les causes de la perte de forêt ou déforestation n'ont pas été abordées dans cette étude. Néanmoins, elles ont été mentionnées dans plusieurs études réalisées en zone tropicale (Puig, 2001; Geist et al., 2002 ; Zang et al., 2002 ; Tsayem et al., 2004, Tsayem, 2008. Scrieciu , 2007 ; Rudel et al., 2009 ; Kissinger et al., 2012 ; Hosonuma et al., 2012; Vermeulen et al., 2011 ; Tchatchou et al, 2015), et plus particulièrement dans la synthèse bibliographique et l'étude des cas des causes de la déforestation dans le bassin du Congo (Gillet et al., 2016).

Il sied également de noter que l'intégration dans la carte finale, des routes extraites du produit de GAF a été faite avec écrasement des valeurs sousjacentes, avant le calcul des statistiques. Cette intégration ne pose pas de problème de cohérence car l'unité minimale de cartographie utilisée dans le produit final ( $0,5 \mathrm{ha})$ est inférieure à celle de GAF (1 ha). Ces routes, qui ont été éditées manuellement dans ce produit avec une forte valeur ajoutée, sont des éléments difficiles à détecter en segmentation automatique. Ces routes correspondent à conversion de la forêt primaire en infrastructure, notamment dans la partie nord du pays où la forêt est concédée aux exploitants forestiers (GAF, 2013). Cette situation a aussi été notée dans une étude récente de changement de couverture forestière dans une zone spécifique du Congo (Bouetou-Kadilamio et al., 2017). Par ailleurs, Megevand et al., (2013) stipulent que si les impacts directs des infrastructures routières sur le couvert forestier sont assez faibles, les impacts indirects et induits constituent eux une menace importante en modifiant la dynamique économique de la zone nouvellement accessible. De nombreuses études rapportent l'impact du développement de routes ou du chemin de fer sur la migration des populations en forêts, la chasse et l'exploitation forestière illégale (Garcia et Feintrenie 
2014 ; Laurance et al., 2014). L'ouverture de routes en forêt tropicale permet le désenclavement et le transport des produits de l'agriculture et des produits issus de la forêt comme le bois, les fruits, le gibier, mais est à l'origine de perte ou de fragmentation des habitats et d'une dégradation environnementale souvent irréversible (Garcia et Feintrenie, 2014 ; Laurance et al., 2014).

\section{Conclusion}

L'approche méthodologique utilisée combinant la télédétection couplée à l'arbre de décision, implémenté dans le langage $\mathrm{R}$ en conjonction avec les outils Open Foris s'est révélée judicieuse dans l'élaboration de la carte de la couverture forestière et des changements pour la période de 2000 à 2012 . Cette carte répond aux critères de la définition nationale de la forêt, adoptés par la République du Congo, pour le calcul du niveau des émissions de référence pour les forêts (NERF) sur la période de référence et constitue une base de données cartographiques importante pour la mise en œuvre des activités REDD+. La carte thématique d'occupation du sol obtenue au cours de nos travaux est acceptable. Elle est de projection sinusoïdale qui est conforme et subit le minimum de déformation en son centre, ce qui convient particulièrement pour le Congo. Les cartes établies par GAF, GFC et FACET de façon indépendante les unes des autres représentent une trame ayant aidé à une meilleure approche méthodologique et à déterminer les pertes réelles de forêt en République du Congo.

Les superficies estimées à travers l'analyse de la précision indiquent que le territoire congolais est dominé par la forêt. Le taux faible de pertes enregistré place la République du Congo dans la catégorie des pays à forte couverture forestière avec un taux faible de déforestation.

\section{Remerciements}

Les auteurs tiennent à remercier vivement tous ceux qui ont, de quelque façon que ce soit, participé à la réalisation de ce travail. Nous pensons notamment aux agents du service de cartographie et photo-interprétation et de la Cellule MNV du CNIAF, et aux représentants du Programme ONUREDD/FAO pour le compte de la République du Congo.

\section{References}

1. Achard, F., Eva, H., Stibig, H. J., Mayaux, P., Gallego, J., Richards, T., and Malingreau, J.P. 2002- Determination of deforestationrates of the world's humid tropical forests. Science, 297: 999-1003.

2. Adjonou K., Ali N., Kokutse. D. A, Novignon S. K. \& Kouami K., 2010. Étude de la dynamique des peuplements naturels de Pterocarpus erinaceus Poir. (Fabaceae) surexploités au Togo. Bois et Forêts des Tropiques, 306: 45-56. 
3. Akadje L. Hauhouot A. C., 2014. Analyse par télédétection environnementales de 2000 à 2013 dans la zone Ramsar de GrandBassam (Sud de la Côte d'Ivoire). Resige, vol. 1, nº 001, 33-40.

4. Bouetou-Kadilamio L, IFO S. et Bisangou S., 2017. Changement de couverture forestière dans le département de la Likouala (République du Congo) durant la période de 1986 à 2015. European Scientific Journal August 2017 edition Vol.13, No.24 ISSN: 1857 - 7881 (Print) e - ISSN 1857- 7431.

5. BRL, 2014. Etude de la spatialisation et de la pondération des causes de la déforestation et de la dégradation forestière et étude sur les options stratégiques REDD+ proposées par le R-PP. Rapport. Ministère de 1'Economie Forestière et du Développement durable.

6. Brown S., 1997. Estimating biomass and biomass change of tropical forest: a primer. FAO Forestry paper 134, Rome, Italy, 55 p.

7. Caloz R.\& Collet C., 2001. Précis de télédétection. Volume 3: traitements numériques d'images de télédétection (Universités francophones). Presses Polytechniques du Québec, Canada.

8. CCNUCC, 2007b. Decision 2/13. Reducing emissions from deforestation in developing countries: approaches to stimulate action. Convention-cadre des Nations Unies sur les changements climatiques, Bonn, Allemagne.

9. <http://unfccc.int/resource/docs/2007/cop13/eng/06a01.pdf>.

10. CCNUCC, 2010. Decision 1/CP.16. Cancun Agreements. Conventioncadre des Nations Unies sur les changements climatiques, Bonn, Allemagne. http://unfccc.int/resource/docs/2010/cop16/eng/07a01.pdf,

11. CCNUCC, 2012. Decision 12/CP.17 Guidance on systems for providing information on how safeguards are addressed and respected and modalities relating to forest reference emission levels and forest reference levels as referred to in decision $1 / \mathrm{CP} .16$ https://unfccc.int/resource/docs/2011/cop17/eng/09a02.pdf CCNUCC, 2013. Decision 13/CP.19. Guidelines and procedures for the technical assessment of submissions from Parties on proposed forest reference emission levels and/or forest reference levels http://www.ciesin.columbia.edu/repository/entri/docs/cop/FCCC_CO P19_dec13.pdf

12. CCNUCC, 2015. Accord de Paris/CoP 21. Convention-cadre des Nations Unies sur les changements climatiques, Paris, France.

13. Chenost C,, Gardette Y-M. ,Demenois J., Grondard N., Perrier M. et Wemaere, 2010. Les marchés du carbone forestier, 167p. 
14. CNIAF (Centre National d'Inventaire et d'Aménagement des ressources forestières et fauniques), 2018. https://github.com/lecrabe/cniaf_2000_2012

15. CNREDD (Coordination Nationale REDD), 2014b. Rapport général de l'atelier national sur la définition " forêt » dans le contexte de la REDD+ en République du Congo dans le cadre du processus REDD+. Brazzaville, $27 \mathrm{p}$.

16. Congalton R. G., 1991. A review of assessing the accuracy of classifications of remotely sensed data. Remote Sensing of Environment, 37: 35-46.

17. Denman, K,L, G, Brasseur, A, Chidthaisong, P, Ciais, P,M, Cox, R,E, Dickinson, D, Hauglustaine, C, Heinze, E, Holland, D, Jacob, U, Lohmann, S Ramachandran, P,L, da Silva Dias, S,C, Wofsy and X, Zhang, 2007, "Couplings Between Changes in the Climate System and Biogeochemistry," In: Climate Change 2007: The Physical Science Basis, Contribution of Working Group I to the Fourth Assessment Report of the Intergovernmental Panel on Climate Change [Solomon, S, D, Qin, M, Manning, Z, Chen, M, Marquis, K,B, Averyt, M,Tignor and H,L, Miller (eds,)], Cambridge and New York: Cambridge University Press.

18. De Wasseige C., Devers D., de Marcken P., Ebal'a Atyi R., Nasi R. et Mayaux Ph., 2009. Les Forêts du Bassin du Congo - Etat des Forêts 2008. Eds : 426 pages, ISBN 978-92-79-132 11-7, doi: 10.2788/32456, Office des publications de l'Union européenne.

19. De Wasseige C. de, de Marcken P., Bayol N., Hiol Hiol F., Mayaux Ph., Desclée B., Nasi R., Billand A., Defourny P., Eba'a Atyi R., 2012, Les forêts du Bassin du Congo - État des forêts 2010, Office des publications de 1'Union européenne, Luxembourg, 276 p. ISBN : 97892-79-22717-2 doi : 10.2788/48830.

20. De Wasseige C., Flynn J., Louppe D., Hiol Hiol F., Mayaux Ph., 2014. Les forêts du bassin du Congo - État des Forêts 2013. Éds. Weyrich. Belgique. 328 p. Dépôt légal : D/2014/8631/30 ISBN : 978-2-87489298-1.

21. Ernst C., Verhegghen A., Bodart C., Mayaux P., de Wasseige C., Bararwandika A., Begoto G., Esono Mba F., Ibara M., Kondjo Shoko A., Koy Kondjo H., Makak J.S., Menomo Biang J.D., Musampa C., Ncogo Motogo R., Neba Shu G., Nkoumakali B., Ouissika C.B. and Defourny P., 2010. Congo Basin forest cover change estimate for 1990, 2000 and 2005 by Landsat interpretation using an automated objectbased processing chain. The International Archives of the Photogrammetry, Remote Sensing and Spatial Information Sciences, XXXVIII-4/C7. 
22. FAO, 2015. Considérations techniques relatives à l'établissement de niveaux d'émissions de référence pour les forêts et/ou niveaux de référence pour les forêts dans le contexte de la REDD+ au titre de la CCNUCC.

23. FAO, 2013. Systèmes nationaux de surveillance des forêts: Surveillance et mesure, notification et vérification (S-MNV) dans le contexte des activités REDD+, $36 \mathrm{p}$. https://www.un-redd.org

24. FACET (Forêts d'Afrique Centrale Evaluées par Télédétection), 2012. Étendue et perte du couvert forestier en République du Congo de 2000 à 2010. Observatoire Satellital des Forêts d'Afrique Centrale (OSFAC). $110 \mathrm{p}$.

25. FRA, 2010, Evaluation des ressources forestières mondiales 2010, Rapport national, Congo, Département des Forêts, Organisation des Nations Unies pour l'alimentation et l'agriculture (FAO), $\mathrm{n}^{\circ} 045$, Rome, Italie, 72p. (www.fao.org/forestry/fra).

26. Garcia C. \& Feintrenie L., 2014. Beyond the mirror: tropical forest fragmentation and its impact on rural livelihoods. In: Kettle C.J. \& Koh L.P. Global forest fragmentation. Wallingford, UK: Cabi, 175-199.

27. GAF, 2013. SGDE Suivi Forestier Extension des Services REDD. S Rapport des opérations de service pour la République du Congo - phase 3.

28. Geist H. \& Lambin E., 2002. Proximate causes and underlying driving forces of tropical deforestation. BioScience, 52(2), 143-150.

29. GFC (2013). http://earthenginepartners.appspot.com/science-2013global-forest

30. GIEC., 2006. Lignes directrices 2006 du GIEC pour les inventaires nationaux de gaz à effet de serre. Vol. 4: Agriculture, foresterie et autres affectations des terres. Hayama, Japon, IGES pour le GIEC (disponible aussi sur: http://www. ipcenggip.iges.or.jp/public/2006gl/ french/index.html), 85p.

31. GIEC, 2007. Changements climatiques 2007. Rapport de synthèse. Résumé à l'attention des décideurs, 114 p. (http://www.ipcc.ch/pdf/assessment-report/ar4/syr/ar4_syr_fr.pdf).

32. Gillet P., Vermeulen C., Feintrenie L., Dessard H. et Garcia C., 2016. Quelles sont les causes de la déforestation dans le bassin du Congo ? Synthès bibliographique et études de cas. Biotechnol. Agron. Soc. Environ. 2016 20(2), 183-194

33. GFOI 2016, Intégration des données de télédétection et d'observation au sol pour l'estimation des émissions et des absorptions de gaz à effet de serre dans les forêts: Méthodes et pratiques recommandées par l'Initiative mondiale pour l'observation des forêts, Version 2.0, 
Organisation des Nations Unies pour l'alimentation et l'agriculture, Rome.

34. Hansen, M. C., P. V. Potapov, R. Moore, M. Hancher, S. A. Turubanova, A. Tyukavina, D. Thau, S. V. Stehman, S. J. Goetz, T. R. Loveland, A. Kommareddy, A. Egorov, L. Chini, C. O. Justice, and J. R. G. Townshend. 2013. "High-Resolution Global Maps of 21stCentury Forest Cover Change." Science 342 (15 November): 850-53. Data available on-line from: http://earthenginepartners.appspot.com/science-2013-global-forest

35. Hewson, J., M.K. Steininger and S. Pesmajoglou, eds. (2014). REDD+ Measurement, Reporting and Verification (MRV) Manual, Version 2.0. Washington, DC, USA. (Manuel de mesure, notification et vérification (MNV) de la REDD+, Version 2.0. Programme Carbone, marchés et communautés, avec le soutien de l'USAID.

36. Hosonuma, N., Herold, M., De Sy, D., De Fries, R.S., Brockhaus, M., Verchot, L., Angelsen, A., Romijn, E., 2012. An assessment of deforestation and forest degradation drivers in developing countries. Environ. Res. Lett. 7, 044009.

37. Karsenty A., Pirard R., 2007b. Changement climatique : faut-il récompenser la « déforestation évitée »? Natures Sciences Sociétés, $\mathrm{n}^{\circ}$ 15 , p. 357-369.

38. Kissinger G., Herold M. \& De Sy V., 2012. Drivers of deforestation and forest degradation - A synthesis report for REDD+ Policymakers. Vancouver, Canada: Lexeme consulting.

39. Laporte N.T., Justice, C., Kendall, J., 1995. Mapping the dense humid forest of Cameroon and Zaire using AVHRR satellite data. International Journal of Remote Sensing, 16 (6) : 1127-1145.

40. Laurance, W.F., Clements, G.R., Sloan, S., O/'Connell, C.S., Mueller, N.D., Goosem, M., Venter, O., Edwards, D.P., Phalan, B., Balmford, A., Van Der Ree, R., Arrea, I.B., 2014a. A global strategy for road building. Nature 513, 229-232.

41. Mapangui, A. and Mpounza M. 2001. "Les sols" in Éditions jeune Afrique \& Menager, Marie-Thérèse \& Lerebours Pigeonnière, Anne \& Mengho, Bonaventure Maurice \& Editions du Jaguar (2001). Atlas du Congo (2e éd). Editions J.A, Paris.

42. Mayaux P., Gond V., Massart M., Pain-Orcet M., Achard F., 2003. Évolution du couvert forestier du bassin du Congo mesurée par télédétection spatiale. Bois et Forêts des Tropiques, ${ }^{\circ} 277$ (3), p.4552.

43. Mayaux P., Janodet E. Blair-Myers C.M., Legeay-Janvier P., 1997. Vegetation Map of Central Africa at 1:5m, TREES Publications Series D1, EUR 17322, Luxembourg: European Commission, 32 p. + 1 map. 
44. Megevand C. et al., 2013. Deforestation trends in the Congo Basin: reconciling economic growth and forest protection. Washington, DC:World Bank. doi: 10.1596/978-0-8213-9827-2. 201pp.

45. Nasi R,, Mayaux P,, Devers D,, Bayol N,, Eba'a Atyi R,, Munier A, Cassagne B, Billand A, Sonwa D., 2008. Un apercu de stock de carbone et leurs variations dans les forêts du Bassin du Congo, pp : 199-2016.

46. NERF, 2017. Niveau des émissions de référence pour les forêts. République du Congo. Rapport, $92 \mathrm{p}$.

47. OFGT, 2018. www.openforis.org

48. Olofsson Pontus, Foody Giles M., Herold Martin, Stehman Stephen V., Woodcock Curtis E., Wulder Michael A. Good practices for estimating area and assessing accuracy of land change. Remote Sensing of Environment 148 (2014) 42-57.

49. Pascal J. P., 2003. Description et Dynamiques des Milieux Forestiers. Notions sur les structures et dynamique des forêts tropicales humides. Rev. For. Fr. LV - numéro spécial 2003: 118 - 130.

50. Pearson T., Harris N., Shoch D., Brown S., 2005. A sourcebook of methods and procedures for monitoring and reporting anthropogenic greenhouse gas emissions and removals associated with deforestation, gains and losses of carbon stocks in forests remaining forests, and forestation. Global Observation of Forest and Land Cover Dynamics, 25p.

51. Puig H., 2001. Diversité spécifique et déforestation : l'exemple des forêts tropicales humides du Mexique. Bois For. Trop., 268(2), 41-55.

52. QGIS Development Team (2018). QGIS Geographic Information System. Open Source Geospatial Foundation Project. http://qgis.osgeo.org

53. R Core Team (2018). R: A language and environment for statistical computing. R Foundation for Statistical Computing,Vienna, Austria. URL https://www.R-project.org/.

54. Ramade F., 2005. Dégradation des écosystèmes et la ruine de la biosphère. In éléments d'écologie, Dunod, Paris, p. 487-516.

55. RPP, 2011. Proposition pour la préparation à la REDD+, République du Congo. Rapport, $213 \mathrm{p}$.

56. Rudel T., Defries R., Asner G.P. \& Laurance W.F., 2009. Changing drivers of deforestation and new opportunities for conservation. Conserv. Biol., 23(6), 1396-1405.

57. Samba G., Nganga D., and Mpounza M., 2007. Rainfall and temperature variations over Congo-Brazzaville between 1950 and 1998. Theor. Appl. Climatol. 91, 85-97 (2008). Springer-Verlag. 
58. SCN, 2009. Seconde communication nationale de la République du CONGO à la Convention-cadre des Nations Unies sur les changements climatiques (CCNUCC). Brazzaville, 190 p.

59. Scrieciu S., 2007. Can economic causes of tropical deforestation be identified at a global level? Ecol. Econ., 62(3-4), 603-612.

60. Snyder, J. P., 1989. Map Projections--A Working Manual. U. S. Geological Survey Professional Paper 1395. Washington, DC: U. S. Government Printing Office, pp. 243-248, 198.

61. Stern Review (Rapport Stern), 2006. L'économie du changement climatique, 34 p., résumé en français diffusé par le journal les Echos. (http://www.lesechos.fr/medias/2007/0115//300131480.pdf).

62. Tchatchou B, Sonwa DJ, Ifo S et Tiani AM. 2015. Déforestation et dégradation des forêts dans le Bassin du Congo: État des lieux, causes actuelles et perspectives. Papier occasionnel 120. Bogor, Indonesie : CIFOR.

63. Tsayem Demaze M., Fosting J.-M. (2004). La déforestation tropicale dans le contexte de mondialisation des risques climatiques et écologiques: outils d'évaluation et de suivi, in (David G., dir.) Espaces tropicaux et risques, du local au global, Presses universitaires d'Orléans et IRD Editions.

64. Tsayem Demaze M., 2008. Quand le développement prime sur l'environnement : la déforestation en Amazonie brésilienne. Mondes Dév., 143, 97-116.

65. Tsayem Demaze M., 2009a. Paradoxes conceptuels du développement durable et nouvelles initiatives de coopération Nord-Sud : le mécanisme pour un développement propre. Article 443, Cybergéo, Revue Européenne de Géographie.

66. Tsayem Demaze M., 2009b. Le protocole de Kyoto, le clivage NordSud et le challenge du développement durable, L'Espace Géographique, $\mathrm{n}^{\circ} 2,2009$.

67. Tsayem Demaze M. ,2011. La télédétection en tant qu'outil de mesure de la réduction de la déforestation (REDD). Revue télédétection, recherche et application, volume 9 (3-4), pp.245- 257. <halshs00576114>.

68. Van der Werf G. R., D. C. Morton, R. S. DeFries, J. G. J. Olivier, P. S. Kasibhatla, R. B. Jackson, G. J. Collatz and J. T. Randerson, 2009. $\mathrm{CO} 2$ emissions from forest loss, Nature Geoscience 2: pp.737-738.

69. Verhegghen A., Defourny P., 2010. A new $300 \mathrm{~m}$ vegetation map for Central Africa based on multi-sensor times series In Third Recent Advance in Quantitative Remote Sensing, J.A. Sobrino (Ed.), Publicaciones de la Universitat de Valencia,Valencia, Spain, 2010. 
70. Verhegghen, A., Mayaux, P., de Wasseige, C. \& Defourny, P., 2012. Mapping Congo Basin vegetation types from $300 \mathrm{~m}$ and $1 \mathrm{~km}$ multisensor time series for carbon stocks and forest areas estimation. In: Biogeosciences, Vol. 9, no. 12, pp. 5061-5079.

71. Vermeulen C. et al., 2011. Enjeux fonciers, exploitation des ressources naturelles et Forêts

des Communautés Locales en périphérie de Kinshasa, RDC. Biotechnol. Agron. Soc. Environ., 15(4), 535-544.

WELD, 2018. http://globalweld.cr.usgs.gov/collections

72. Zhang, Q., Justice, C., Desanker, P., Townshend, J., 2002. Impacts of simulated shilfting

cultivation on deforestation and the carbon stocks of the forests of Central Africa. Agriculture. Ecosystems \& Environment, 90(2):203209. 